Hydraulic Engineering Repository

Ein Service der Bundesanstalt für Wasserbau

Marsili, Francesca; Bödefeld, Jörg; Croce, Pietro; Landi, Filippo

Bayesian approaches to lifetime prediction

Originalveröffentlichung / Original Publication:

http://library.oapen.org/handle/20.500.12657/30004

Verfügbar unter / Available at:

https://hdl.handle.net/20.500.11970/108288

Vorgeschlagene Zitierweise / Suggested citation:

Marsili, Francesca; Bödefeld, Jörg; Croce, Pietro; Landi, Filippo (2018): Bayesian approaches to lifetime prediction. In: Safety and reliability - safe societies in a changing world, proceedings of the 28th International European Safety and Reliability Conference (ESREL 2018), Trondheim, Norway, 17-21 June 2018 (): Taylor \&amp; Francis Group. S. 707-715. http://library.oapen.org/handle/20.500.12657/30004. 


\title{
Bayesian approaches to lifetime prediction
}

\author{
F. Marsili \& J. Bödefeld \\ Federal Waterways Engineering and Research Institute, Karlsruhe, Germany \\ P. Croce \& F. Landi \\ Department of Civil and Industrial Engineering, University of Pisa, Italy
}

\begin{abstract}
In this paper a framework to predict the remaining lifetime for existing waterways infrastructures based on stochastic modeling of deterioration processes and Bayesian analysis is presented. The application of the Bayes' theorem is motivated by the availability of expert knowledge as well as the collection of both qualitative and quantitative data from the structure. An original method is proposed to derive the prior statistical parameters of the gamma distribution describing the stochastic deterioration process, based on the assumption that the lifetime distribution can be approximated by the Birnbaum-Saunders statistical model. An appropriate Bayesian Network is finally implemented to improve the classification of the structure with respect to its proneness to damage. The outcome of the research work is to assist the owners of large infrastructural network in planning and prioritizing maintenance interventions.
\end{abstract}

\section{INTRODUCTION}

In the last decade the management of assets of infrastructures is becoming an increasingly important engineering task. As it is also stated in ISO 55000 (2014), accurate prediction of lifetime distributions are required to develop strategic asset management plan, and the use of a life-cycle management approach is fundamental to realize value from the asset. The German Federal Waterways Engineering and Research Institute (BAW) has developed a management system based on fixed inspection intervals, allowing, in principle, to optimize the repair or strengthening interventions, which are planned and carried out according to the outcomes of the inspections themselves. Although plenty of data regarding structures' condition are available in BAW, further steps should be undertaken in order to extract information regarding asset's lifetime (Haider 2012). The transformation of data into useful information is not always straightforward, and several approaches could be found in literature: for example, Trappey (2012) evaluates the asset lifetime using logistic regression; Lim \& Mba (2013) estimate the remaining useful life implementing Kalman filter, while Tse \& Shen (2013) pursue the same scope using support vector machines.

Another way to reach this goal is through a more accurate lifetime prediction, which could be obtained by modelling degradation phenomena through stochastic processes (Riascos-Ochoa 2016); the parameters of the process can be also updated applying the Bayes' Theorem, given any new information about the state of damage (Ang $\&$ Tang 2007). However this approach, which has already been adopted by Bousquet (2014) and Haowei (2015), has the disadvantage that only quantitative data can be considered for the updating, and only the uncertainty directly affecting model parameters can be reduced.

This paper thus proposes a new procedure, which allows updating prior knowledge considering both quantitative data and qualitative information through the definition and the implementation of a Bayesian Network. Besides, using the Bayesian Network allows also identifying the stochastic process better describing the degradation phenomena affecting the structures; the Bayes' Theorem is thus applied in order to reduce the uncertainty affecting the parameters of the identified stochastic process.

The paper is subdivided in the following way: in Chapter 2 the current approach to the asset management implemented by the BAW is presented, focusing the attention on its advantages and drawbacks; in Chapter 3 the available information for lifetime prediction is examined; in Chapter 4 the new approach to lifetime evaluation is presented, also briefly reviewing the theoretical background to gamma processes, Bayesian Analysis and Bayesian Network; in Chapter 5 the new approach is applied to a real case study, paying particular attention to the elicitation of the prior distribution and the Bayesian Network; in Chapter 6 conclusion and outlook are drawn. 


\section{CURRENT APPROACH TO ASSET MANAGEMENT}

The BAW has developed tools for the management of a huge number of waterways infrastructures. The entire portfolio comprises several types of construction such as locks, weirs, culverts, canal bridges and lighthouses, also having different ages. A huge part of the asset is represented by massive structures older than 100 years and designed according to empirical methods with limited and simplified static calculations. These structures are characterized by sections with a large thickness, in which a multi-axial stress state takes place. Therefore they exhibit significant "reliability reserves" and relevant plastic resources, as demonstrated by the low number of recorded collapses which were mainly characterized by ductile failure modes. Despite their satisfactory performance at the ultimate limit states, these structures often fail to meet serviceability requirements such as crack width limitations or deformations (BAW 2015). Furthermore, several other time-dependent factors may affect their service life, also depending on climate change (Orcesi 2016) and obsolescence (Langston 2011). But, above all, main degradation phenomena affecting such kind of structures are spalling and corrosion. In order to manage maintenance intervention, the BAW developed an ad hoc maintenance management system called EMS-WSV (Bödefeld \& Kloè 2012), shortly summarized in the following.

A database software, called WSVPruf, is used to store data collected on each structure during execution, inspection and maintenance. All damages are rated on an increasing scale from level 1 to level 4 (1: good condition; 4: critical condition) and they are recorded in a standard format by the program. Another program called 'Zustandsprognose' forecasts future deterioration stages of the structure for the next 20-30 years. Here, different approaches have been considered:

- Survival functions are applied to describe the deterioration of components where no evident damage is detected at the actual inspection time;

- A method based on discrete-time Markov processes is implemented in order to forecast the deterioration of detected damages. The parameters of the transition matrix are also determined according to survival functions;

- In some cases, physical equations have been used in order to validate Markov Chains.

Once the survival functions and Markov Chains have been defined, the evolution of the damage scale in time can be determined in an almost deterministic way, as a unique process. The remaining lifetime is also expressed in a deterministic way, and it represents the time lapse interval required to the damage scale in order to reach the critical condition, corresponding to level 4.

Obviously, the above mentioned methods present some advantages and drawbacks. On one hand, physical equations are usually considered as deterministic; they model only some deterioration processes and they require a huge amount of data to determine the main parameters of the physical laws. On the other hand, survival functions and Markov chains are powerful and flexible methods and they can be easily adapted to different deterioration processes. But, in both cases, it is difficult to take into account the influence of different factors on future degradation stages. In case of Markov Chains, the state of the structure is described through a unique variable, and there are no consolidated methods according which the parameters of the transition matrix can be defined. It must be also underlined that degradation phenomena largely depend on other factors such as the environmental condition and the quality of the materials, which is mainly the quality of concrete. Anyhow, despite they are crucial to determine the proneness to damage of the structure and to predict the remaining lifetime, these factors are almost completely disregarded by the current approach.

\section{AVAILABLE INFORMATION FOR LIFETIME PREDICTION}

Whether survival functions of Markov Chains are implemented, the parameters of the models should be determined from real data obtained from inspections, surveillance or ad hoc investigations. Obviously, provided that enough data exist, the parameters can be obtained through some sort of regression analysis or statistical investigation; but, since inspections are usually carried out every six years, surveillance is executed not later than three years after each principal inspection, and ad hoc inspections are only required after accidental shocking events such as ship impacts or flooding, available data are often not sufficient.

As underlined also by Haider (2012), asset lifecycle management is an information intensive task that requires generating, processing and analyzing huge amount of information. Data usually consist in both qualitative and quantitative information, able to describe the static, constructional and hydromechanical condition of the structure; however quantitative data collected during the inspections, being mainly obtained through simple measuring instruments, are quite rough, and for this reason they are affected by great uncertainties. 
Another source of information is represented by expert knowledge. In the present case, this information was previously collected through a Delphi Interview submitted to a total amount of 28 experts (BAW 2009). In the Delphi Interview it was asked to answer the following question: "When does a special damage appear for the first time in your opinion?", or in other words, it was asked to elicit the survival functions given a certain degradation process and a specified degradation level. Three degradation levels were especially considered, notionally corresponding to damage levels 2 , 3 and 4, subdividing the asset into three categories: fragile, normal and robust constructions, and a choice sheet with several different predefined time intervals (decades) was provided to the experts to facilitate the comparison of the answers.

Other relevant information can be also extracted by unstructured data and secondary database of tests results by conducting data analysis with suitably developed algorithms and software, as shown by Gao \& Koronios (2012) and Croce (2018). This information can be supplemented by the results of ad hoc investigation such as material or chemical tests carried out on the considered structure or on similar constructions, built in in the same time period in a given geographical area, adopting comparable construction techniques.

Finally, data need to be acquired about relevant climatic actions influencing some degradation processes, like temperature or moisture, as well as about effects of climate change on them.

Aleatoric and epistemic uncertainties are inevitably associated to the above mentioned information, the second one representing a lack of knowledge, which can be reduced as soon as further data become available.

\section{A NEW APPROACH TO LIFETIME PREDICTION}

\subsection{Description of the new procedure}

In order to obtain more realistic lifetime prediction, an innovative method is proposed in the paper, where the deterioration phenomenon is modelled by an appropriate stochastic process, a gamma process, while epistemic uncertainties are suitably reduced resorting to the Bayesian Theorem.

Although updating the parameters of a stochastic process through data collected during inspection was already suggested (Bousquet 2014, Haowei 2015), the issue here presented involves some complications that have not been yet considered. One complication is due to the fact that different levels of epistemic uncertainties affect the lifetime prediction. A first level is connected to the proneness of the structure to damage: in fact, even if three categories of constructions can be defined (fragile, normal and robust) according to Chapter 3, the "category" to which the structure belongs is not known a priori. A second level is represented by the uncertainty affecting the parameters of the gamma process describing the degradation phenomenon within each category.

Moreover, as information derived from the structure is both qualitative and quantitative, but the degradation phenomenon is mainly described in quantitative terms, the problem becomes more and more complex. Nevertheless, qualitative data are important in order to figure out the sensitivity of the structure to damage, and the variables better describing the degradation process. Thus the question becomes: how could qualitative and quantitative data be considered in order to reduce the two levels of epistemic uncertainties previously identified?

A possible answer to this question is to resort to a Bayesian Network (BN) and to use it as a Naïve Bayesian Classifier in order to identify the "Proneness to damage" category of the structure and to remove the first level of epistemic uncertainty. Once the category has been identified, the parameters of the gamma process describing the degradation phenomenon within that class could be further updated considering the data about the damage progression collected during the inspection on the structure.

In the following, the theoretical background to the proposed approach is briefly introduced.

\subsection{Gamma process}

Gamma process is always applicable to model positive and strictly increasing degradation data, as suggested by Nicolai (2007) and van Noortwijk (2009).

A non-stationary gamma process $Y(t)$ with shape function $\Lambda(t)>0$ and scale parameter $\beta$ has the following properties:

1. $Y(0)=0$ with probability one;

2. $Y(\tau)-Y(t) \sim \Gamma(\Lambda(\tau)-\Lambda(t), \beta), t \in[0, \tau)$;

3. $Y(t)$ has independent increments;

where $\Gamma($.$) is the gamma function. Conditions (1)$ and (2) provide the probability density function of a gamma process:

$$
f(Y, \Lambda(t), \beta)=\frac{\beta^{-\Lambda(t)}}{\Gamma(\Lambda(t))} Y^{\Lambda(t)-1} \exp \left(\frac{-Y}{\beta}\right) .
$$

Thus the expected deterioration at time $t$ can be expressed by a power law:

$$
E(Y(t))=\frac{\Lambda(t)}{\beta}=\frac{\alpha t^{c}}{\beta},
$$


where $\alpha / \beta>0$ and $c>0$ is a parameter describing the shape of the expected deterioration. Values of the parameter $c$ for some relevant deterioration processes are given by van Noortwijk (2009).

Suppose now that the lifetime $\xi$ is defined as the time when $Y(t)$ reaches a suitable failure threshold $D$ (first passage). Then the cumulative distribution function (CDF) of $\xi$ can be obtained as:

$$
F(t)=\frac{\Gamma\left(\Lambda(t), D_{\beta}\right)}{\Gamma(\Lambda(t))}
$$

where $D_{\beta}=D / \beta$. Park \& Pedgett (2005) showed that the exact probability distribution function $(p d f)$ of $\xi$ for a gamma process can be extrapolated from Equation (3). However, since the resulting expression is too complex for practical application, they proposed to approximate the CDF of $\xi$ with the Birnbaum-Saunders (BS) distribution (Birnbaum \& Saunders 1969), so that the mean lifetime $\bar{\xi}_{B S}$ simply results:

$\bar{\xi}_{B S}=\left[D \frac{1}{\alpha \beta}+\frac{1}{2 \alpha}\right]^{c}$.

\subsection{Bayesian updating}

As known, Bayes' theorem represents an actualization principle and it allows the calculation of conditional probabilities or conditional densities.

In the discrete case, it describes the updating of $p\left(A_{i}\right)$ to $p\left(A_{i} \mid B\right)$ once observed the event $B$.

In the continuous case, given two random variables $X$ and $Z$, with conditional distribution of $X$ given $Z f(x \mid z)$ and marginal distribution $g(z)$, it describes the conditional distribution of $Z$ given $X$ :

$$
f(z \mid x)=\frac{f(x \mid z) g(z)}{\int f(x \mid z) g(z) d z}
$$

Depending on the interpretation of probability, the meaning of Bayes' theorem differs significantly. If probability (or density) is interpreted in a frequentistic manner, the theorem expresses the proportion of an event given the occurrence of another event. Conversely, if probability reflects the relative plausibility or degree of belief attributed to a certain event, Bayes' theorem forms the mathematical basis for adjusting or updating the probability as soon as more evidence becomes available.

Also the uncertainty on the parameters $\theta$ of a model could be described by a probability distribution, which has to be interpreted in most of the cases as a degree of belief. If the model is represented by a density function, a two-levels hierarchical model can be obtained, in which the second level is represented by the $p d f$ on the statistical parameters of the probability model at the first level. The statistical parameters of the second level distribution are usually called 'hyperparameters', and the $p d f$ 'hyperdistribution'. Anyhow, the degree of belief can be updated when new data are available, so that, once defined the posterior distribution, statements about the parameter can be made.

The computation of the posterior distribution involves the calculation of several integrals and for this reason is not straightforward. Simplified approaches have been sought in order to facilitate the updating of the prior distribution. The most popular approach is to resort to the so called conjugate prior distributions, which have the appealing features that prior and posterior distributions have the same functional form, and the updated parameters can be computed in an analytical way. In case of the gamma distribution, the conjugate prior distribution is characterized by rate parameter $\theta=1 / \beta$ following a gamma distribution, denoted as $\theta^{\prime} \sim \Gamma\left(a^{\prime}, v^{\prime}\right)$ where $v^{\prime}=1 / b^{\prime}$ is the rate parameter and $b^{\prime}$ is the scale. Let $\boldsymbol{x}=\left[x_{0}, x_{1}, \ldots, x_{n}\right]$ the observed degradation data, $t=\left[t_{0}, t_{1}, \ldots, t_{n}\right]$ the corresponding times; denoting with $\Delta x_{i}=x_{i}-x_{i-1}$ and $\Delta t_{i}=t_{i}-t_{i-1}$ the degradation and the time increments, respectively, the posterior shape and rate parameter for $\theta$ can be computed in the following way (Ang \& Tang 2007):

$$
\begin{aligned}
& a^{\prime \prime}=a^{\prime}+\alpha\left(t_{n}-t_{0}\right) \\
& v^{\prime \prime}=v^{\prime}+x_{n}-x_{0}
\end{aligned}
$$

and the posterior mean of $\theta$ can be written as:

$$
E(\theta \mid \Delta x)=\frac{\varepsilon^{\prime \prime}}{v^{\prime \prime}} .
$$

from which the scale parameter $\beta$ is easily obtained.

\subsection{Bayesian Network}

A Bayesian Network (BN) is a flexible tool that allows a rigorous processing of both quantitative and qualitative information (Kjærulff \& Madsen 2008). It is defined as a directed acyclic graph (DAG) which determines a factorization of a joint probability distribution, as the nodes of the DAG represent the variables and the directed links describe the factorization. For each direct link from a node $X$ to a node $Y$ (where $X$ is here the 'parent' and $Y$ the so-called 'child'), a conditional probability $p(Y \mid X)=z$ is attached to $Y$. The conditional probability expresses a rule that assumes the following form: if $X=x$ then $Y=y$ with probability $z$, where $y$ and $x$ denote the state of $Y$ and 
$X$, respectively. Usually $Y$ represents the effect of $X$, typically not observable by itself, but whose state is inferable via prior $p d f p(X)$, conditional $p d f$ $p(Y \mid X)$ and Bayes' theorem:

$$
p(X=x \mid Y=y)=\frac{P(Y=y, X=x)}{\sum_{X} P(Y=y, X=x)}
$$

Observation may have the form of hard evidence if zero probability is assigned to all but one state; otherwise it is said to provide soft evidence. So far it is implied that the nodes of the $\mathrm{BN}$ represent discrete variables, at which probability tables expressing conditional probabilities are attached. However it is also possible to have continuous variables: in this case it is necessary to specify a density function for each combination of states for the parent variables. Whether discrete or continuous variables, $\mathrm{BN}$ can be applied to solve a wide range of problems, and, inter alia for classification purposes. In this specific case, they are especially called Naïve Bayesian Classifier, because of the strong (naïve) independence assumptions assumed among the features that determine the class label drawn.

\section{CASE STUDY}

In order to clarify the proposed approach, a case study is here developed. The attention is mainly focused on the elicitation of the prior gamma distribution describing the stochastic process; this task is carried out exploiting the approximation of the lifetime distribution with the BS distribution. The case study aims to illustrate a practical application of the proposed method referring to a unique damage detected on an existing lock. Extensions to cases involving several damages and different deterioration processes will be discussed in future works.

\subsection{Elicit the prior distribution}

A prior distribution for the model parameters is elicited according to the expert knowledge collected through the Delphi interview described in Chapter 3. It would be easier if experts could have been asked to represent their opinion in statistical terms, for example: "Given the degradation phenomena modelled through a gamma process, what would be the shape and the scale parameters of the gamma distribution?". However it is unlikely that experts could give an accurate answer. Except in case of symmetric distribution, when mean value and standard deviation can be easily elicited, they usually think in terms of percentile. Moreover, expert knowledge actually concerns expected lifetimes rather than damage increments; for this reason, the prior information should be transformed in a form leading to an easy identification of the prior gamma distribution. One way to do this is by using Equation (4) which links the parameters of the gamma distribution with the expected lifetime. Given the expected lifetime required to reach certain degradation levels, and the speed of the process, represented in some way by the value of $c$, the parameters of the gamma distribution can be easily obtained by solving the system of equations. In this case, two equations are required to determine the two parameters: those related to damage levels 2 and 3 are considered, as expert knowledge about events that happened earlier should be more reliable compared to that related to future events. The uncertainty on the parameter is similarly defined, considering the uncertainty affecting the expected lifetimes and assuming a confidence interval. The procedure, which is very general, can be applied to elicit prior distribution for any degradation process.

In the specific case, in Table 1 the average of the expert predictions are summarized in terms of time lapses required by the crack width to reach the levels corresponding to different degradation class, while in Table 2 the confidence intervals of the predicted time lapses, corresponding to probability of exceedance of $75 \%$ and $25 \%$, respectively are reported. Given the progress in time of the degradation process, it is also possible to conclude that the degradation speed can be assumed constant, at which $c=1$ corresponds. For example, in case of fragile construction, the system of equations results:

$$
\left\{\begin{array}{l}
10=\frac{0.3}{\alpha \beta}+\frac{1}{2 \alpha} \\
20=\frac{0.7}{\alpha \beta}+\frac{1}{2 \alpha}
\end{array}\right.
$$

The values of parameters $\alpha$ and $\beta$ obtained by solving all the systems of equations are shown in

Table 1. Expert knowledge results-Delphi Interview - (DR: damage level, CW: crack width, $\bar{\xi}$ : expected lifetime, F: fragile, N: normal, R: robust).

\begin{tabular}{llllr}
\hline & & \multicolumn{3}{c}{$\bar{\xi}$ (years) } \\
\cline { 3 - 5 } DL & CW & F & N & R \\
\hline 2 & 0.3 & 10 & 25 & 50 \\
3 & 0.7 & 20 & 50 & 100 \\
4 & 1 & 30 & 75 & 150 \\
\hline
\end{tabular}


Table 3, while the simulation of the gamma process for fragile, normal and robust structures with these values are shown in Figures 1, 2 and 3, respectively.

For analytical and mathematical tractability, it is then assumed that only $\beta$ is random and it also follows a gamma distribution, so that the prior distribution is then conjugate through the likelihood function to the posterior, simplifying the updating calculation. The prior distribution can be defined by considering the uncertainty affecting the expected lifetime: each time lapse interval corresponds to an interval for the parameter $\beta$. Assuming the same probabilities of exceedance, it will be possible to elicit the shape $a^{\prime}$ and scale $\mathrm{b}^{\prime}$ (or the rate $v^{\prime}$ ) parameters of the prior distribution (Table 3 ).

\subsection{Elicit the Bayesian Network}

The Bayesian Network is elicited according to prior information and the previously defined gamma process. In the remainder of the paper, only the most fundamental variables will be considered; anyhow, as soon as that further analysis will be carried out, it will be possible not only to consider the relationships among a greater number of variables, but also to elicit the structure of the network and the conditional probabilities from data itself rather than from expert knowledge. The variables that will be considered are: the proneness to damage of the structure (also called damage category (DC), characterized by three possible states: Fragile (F), Normal (N) and Robust $(\mathrm{R}))$, the concrete quality $(\mathrm{CQ}$, characterized by three possible states: $\operatorname{Bad}(\mathrm{B})$, Normal (N), Good (G)), the damage quantity $(\mathrm{DQ}$, characterized by three possible states: extended (E), limited (L), sporadic (S)) and

Table 2. Confidence intervals for the lifetime $\xi$ predicted by the experts (F: fragile, N: normal, R: robust).

$$
\xi_{25 \%}-\xi_{75 \%} \text { (years) }
$$

\begin{tabular}{rlc}
\hline $\mathrm{F}$ & $\mathrm{N}$ & $\mathrm{R}$ \\
\hline $8-12$ & $20-30$ & $40-60$ \\
$16-24$ & $40-60$ & $80-120$ \\
$24-36$ & $60-90$ & $120-180$ \\
\hline
\end{tabular}

Table 3. Elicited parameters of gamma process and statistical parameters of the gamma distribution describing the uncertainty over the scale $\beta$.

\begin{tabular}{llllllll}
\hline & $\alpha$ & $\beta_{50 \%}$ & $\beta_{25 \%}$ & $\beta_{750 \%}$ & $\mathrm{a}^{\prime}$ & $\mathrm{b}^{\prime}$ & $\mathrm{v}^{\prime}$ \\
\hline $\mathrm{F}$ & 0.2 & 0.2 & & & & & \\
$\mathrm{~N}$ & 0.08 & 0.2 & 0.15 & 0.25 & 7.27 & 0.75 & 1.33 \\
$\mathrm{R}$ & 0.04 & 0.2 & & & & & \\
\hline
\end{tabular}

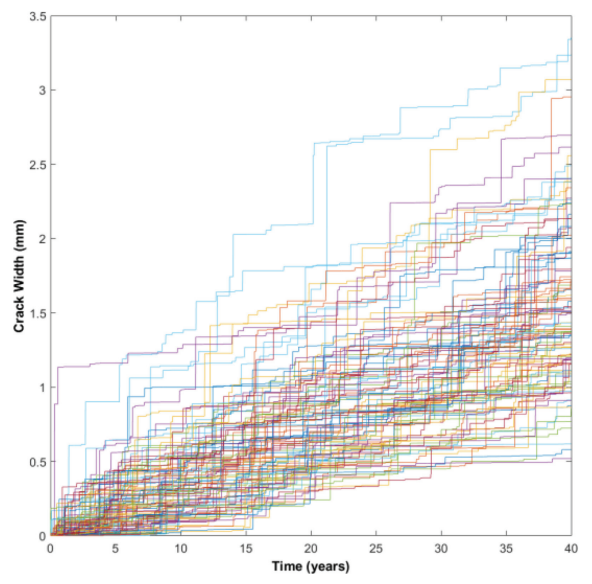

Figure 1. Simulation of the gamma process for fragile structures (100 sample paths).

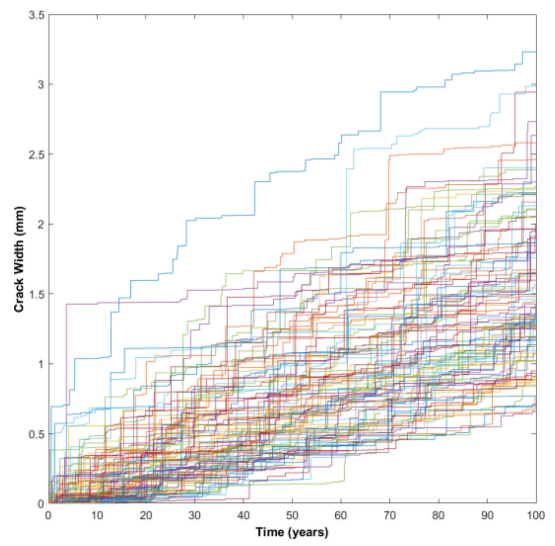

Figure 2. Simulation of the gamma process for normal structures (100 sample paths).

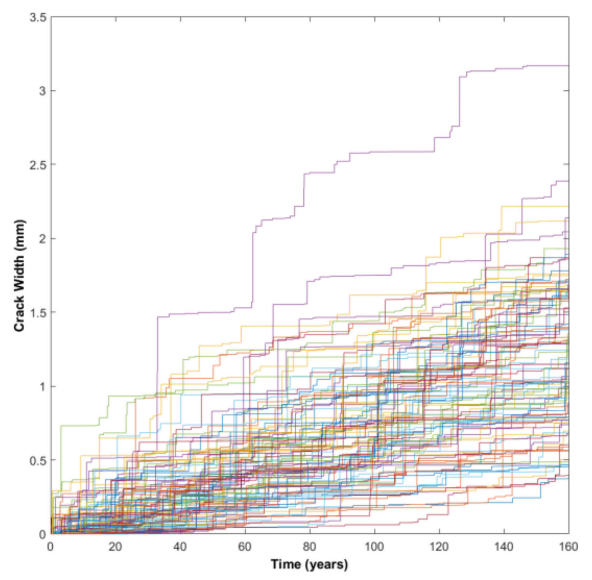

Figure 3. Simulation of the gamma process for robust structures (100 sample paths). 


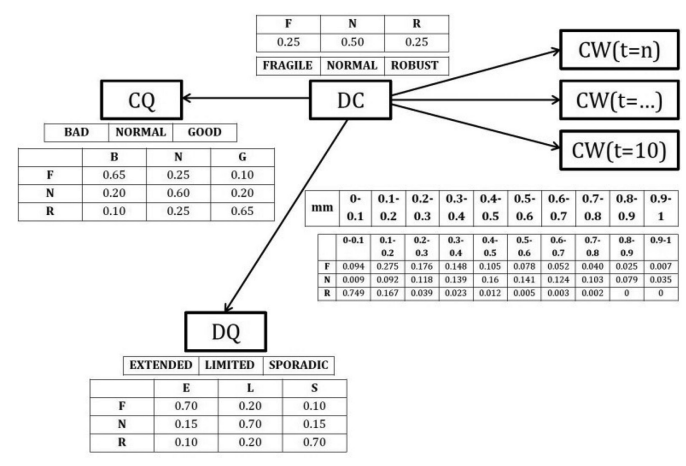

Figure 4. Bayesian Network implemented in order to classify the structure with respect to its proneness to damage.

the crack width referred to different time steps $(\mathrm{CW})$, characterized by 10 possible states.

The states of each variable, the dependency relationships and the associated probability tables are shown in Figure 4. For the sake of simplicity only the probability table referred to $t=10$ years is here showed.

The concrete quality, the damage quantity and the crack width can be observed, while the updating of the probability of the damage categories, that cannot be observed, is a key objective. It is assumed that the observable variables are independent, given the variable that it should be classified. Although this assumption facilitates calculation of posterior probabilities, it is actually inaccurate, as it is often the case with Naïve Bayesian Classifier. Nonetheless it has been demonstrated that the crude independence model could perform surprisingly better than more complicated alternatives (Hand 2001); furthermore precise estimation for the posterior class probabilities are here not required, since they will be used for comparison purposes, as better clarified in Chapter 5.3. By collecting evidence regarding the observable variables, it will be possible to infer the proneness to damage of the structure. It is important to underline that assuming a parametric deterioration process simplifies the relationships among the variables; in effect, the deterioration at instant $t$ can be considered independent from the deterioration at the previous instant, preventing us to resorting to a much complicated dynamic Bayesian Network (Straub 2009, Ramìrez \& Utne 2015). Furthermore, the probability table associated to the variable 'crack width' can be easily obtained simulating the gamma process. The variable is actually continuous, and the degradation at each time step $t$ is represented by a gamma distribution with shape $\alpha t$ and scale $\beta$ (see Chapter 4.2). However BNs that involve continuous random variables imply some complications, which are outside the purposes of the present paper and will be discussed later in further studies. Moreover, as it can be seen later from Table 4, data about crack widths are actually given in terms of intervals rather than single values, since, as already stressed, the measuring devices are rough and measures are inevitably affected by great uncertainty. Also for this reason, the continuous variables are then discretized so that each state corresponds to collectable measures.

\subsection{Implementation of the Bayesian Network and updating of the gamma process}

Data referring to three different structures (A, B and $\mathrm{C}$ ) and related to a damage event having a single cause are considered in the case study (Table 4).

First of all the data are used in order to classify the structure by using the previously defined $\mathrm{BN}$. If all the data point out that one category has very high updated probability (for example higher than 0.70 ), then it is possible to directly consider the Gamma distribution corresponding to that category and the related uncertainty of the statistical parameters for a further Bayesian updating. However, in several cases, the results could not point out clearly at a certain category, because two adjacent categories have similar updated probabilities; in this case, it is possible to conservatively consider only the lowest category, or to collect further data.

Once that the prior model will be chosen, it is possible to update it resorting to the analytical formula expressed by Equations (6) and (7).

Table 4. Inspection results on three structures, A, B, C, (CQ: concrete quality, DQ: damage quantity, CW: crack width).

\begin{tabular}{|c|c|c|c|c|c|c|c|c|}
\hline \multirow[b]{2}{*}{ Str. } & \multirow[b]{2}{*}{ Year } & \multirow[b]{2}{*}{ CQ } & \multicolumn{3}{|c|}{ 1st inspection } & \multicolumn{3}{|c|}{$2^{\circ}$ inspection } \\
\hline & & & Year & DQ & CW & Year & DQ & $\mathrm{CW}$ \\
\hline A & 1995 & 1 & 2005 & 1 & 0.5 & 2017 & 1 & 0.8 \\
\hline B & 1975 & 2 & 2005 & 2 & 0.5 & 2017 & 2 & 0.7 \\
\hline $\mathrm{C}$ & 1955 & 3 & 2005 & 3 & 0.3 & 2017 & 3 & 0.4 \\
\hline
\end{tabular}

Table 5. Updated probabilities for damage category (DC) derived from the Bayesian Network, updated statistical parameters for gamma process, prior and updated lifetime prediction $\bar{\xi}$ in years, obtained simulating the gamma process.

\begin{tabular}{llllllllr}
\hline & $\mathrm{F}$ & $\mathrm{N}$ & $\mathrm{R}$ & $\varepsilon^{\prime \prime}$ & $\mathrm{v}^{\prime \prime}$ & $\overline{\beta^{\prime \prime}}$ & $\overline{\xi^{\prime}}$ & $\bar{\xi}^{\prime \prime}$ \\
\hline 1 & 0.96 & 0.03 & 0.01 & 9.67 & 1.62 & 0.16 & 28 & 33 \\
2 & 0.07 & 0.72 & 0.21 & 8.23 & 1.52 & 0.184 & 65 & 70 \\
3 & 0.01 & 0.09 & 0.90 & 7.75 & 1.42 & 0.183 & 135 & 140
\end{tabular}


Finally $\beta^{\prime \prime}$ can be used to improve the prediction of the structure lifetime when the limit damage level will be reached; the prediction can be obtained by simulating the gamma process considering the updated parameter.

The results are listed in Table 5.

\section{CONCLUSION}

An innovative procedure to lifetime prediction exploiting the potentialities of the Bayes' Theorem has been proposed, as depicted in the flowchart shown in Figure 5, based on the following points:

- The degradation phenomenon is modelled as a stochastic process in which degradation increments are gamma-distributed.

- Considering background information and qualitative and quantitative data collected during inspections, a Bayesian Network is adopted to classify the proneness to damage of the structure.

- Uncertainties affecting the parameters of the stochastic process are updated considering quantitative data about the degradation level reached by the structure by applying the Bayes Theorem.

Advantages of the proposed method are:

- Qualitative and quantitative data can be used at the same time, to refine lifetime predictions.

- Adoption of gamma processes to model degradation phenomena simplifies the structure of the Bayesian Network and the computation of the conditional probabilities.

A case study has been finally developed in order to show a practical application of the proposed method. Attention has been largely devoted to the elicitation of the prior distribution for the gamma process, and real data collected during inspection on real structures have been considered. In authors' opinion, this approach is very promising although some aspects need further studies. In effect, the value of the constant $c$, that regulates the speed of degradation, should be determined more precisely, also considering different degradation phenomena. An improved Bayesian Network including continuous variables, representing the state of damage at different time steps and whose parameters are directly derived from measurements, should be suitably developed. Uncertainties in the collected data, as well as uncertainties in the degradation limits should be duly considered too. At the final stage, once the proposed procedure is improved and suitably validated, its efficiency should be also verified by comparing theoretical time lapse predictions with empirical data on a sufficiently large number of existing structures. The approach can be implemented in order to plan in a

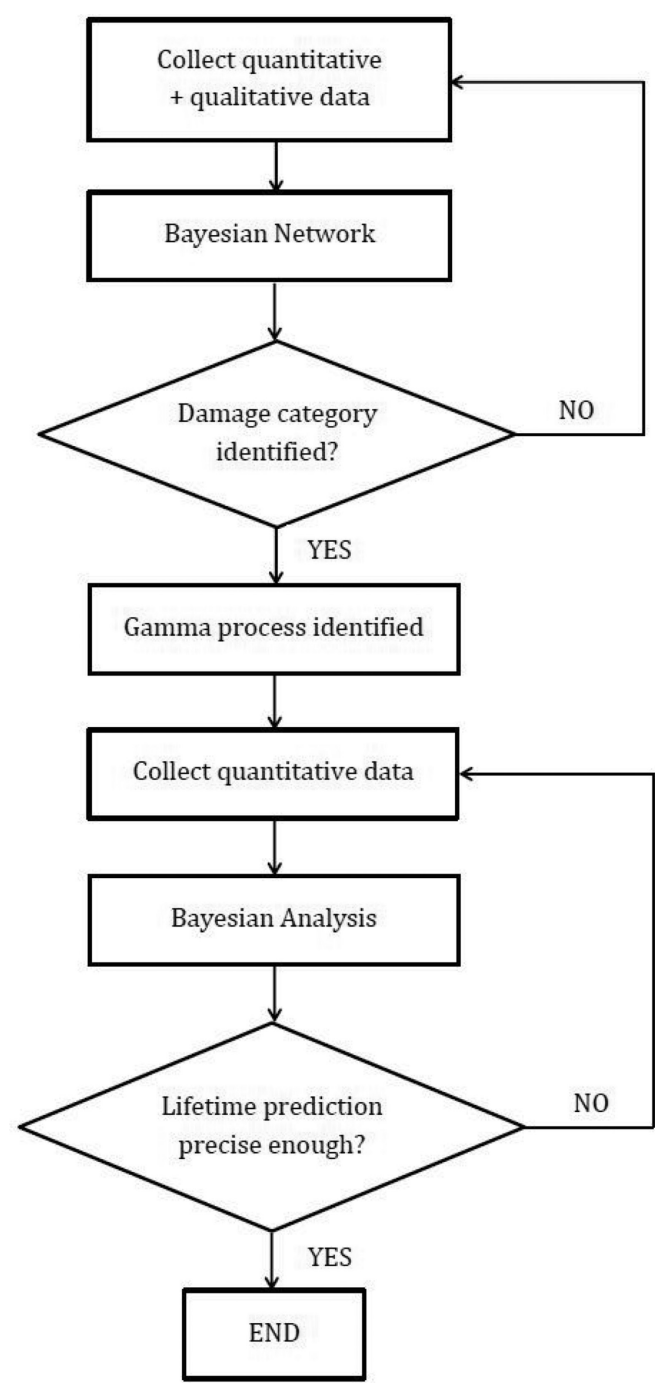

Figure 5. Flowchart summarizing the proposed procedure.

more precise and realistic way asset life-cycle, and to identify on which structures maintenance interventions should be firstly performed.

\section{REFERENCES}

Ang, H.S. \& Tang, W.H. 2007. Probability Concepts in Engineering: Emphasis on Applications to Civil and Environmental Engineering, 2nd Ed. Hoboken, NJ, J. Wiley \& Sons.

BAW 2009. Erhaltungsmanagementsystem für die WSV, Phase 2 - Meilensteinbericht 2009 (internal report). 
BAW 2015, Schadensklassifizierung an Verkehrswasserbauwerken (MSV), www.baw.de.

Birnbaum, Z.W. \& Saunders, S.C. 1969. A new family of life distributions, J. of Appl. Prob., 6, 2, 319-327.

Bödefeld, J. \& Kloè, K. 2012. Managementsystem for infrastructure of waterways, 3rd Int. Symp. on Lyfe Cycle Eng.

Bousquet, N. Fouladirad, M. Grall, A. Paroissin, C. 2015. Bayesian gamma processes for optimizing conditionbased maintenance under uncertainty, Appl. Stoch. Mech. Bus. Ind., 31 360-379.

Croce, P. Marsili, F. Klawonn, F. Formichi, P. Landi, F. (2018), Evaluation of statistical parameters of concrete strength from secondary experimental test data, Construction and Building Materials, 163, 28, 343-359.

Gao, J. \& Koronios, A. (2012), Unlock the Value of Unstructured Data in EAM. Proc. 7th W. Cong. Eng. Asset Man.

Haider A. (2012), Asset Lifecycle Data Governance Framework, Proc. 7th W. Cong. on Eng. Asset Management.

Hand, D.J. \& Yu, K. (2001), Idiot's Bayes-not so stupid after all?, International Statistical Review. 69 (3): 385-399.

Haowei, W. Tingxue, X. Qiaoli, M. 2015. Lifetime prediction based on Gamma processes from accelerated degradation data, Chinese Journal of Areonautics, 28(1): 172-179.

ISO 55000, Asset management-Overview, principles and terminology, 2014.

Kjærulff, U.B. \& Madsen, A.L. (2008). Bayesian Networks and Influence Diagrams, Springer.

Langston, C. (2001), Estimating the useful life of buildings, Conference Papers, Paper 30.

Lim, R. \& Mba, D. (2013), Fault Detection and Remaining Useful Life Estimation Using Switching Kalman
Filters, Eng. Asset Management-Systems, Professional Practices and Certification, Lecture Notes in Mech. Eng.

Nicolai, P. Dekker, R. van Noortwijk, J.M. 2007. A comparison of models for measurable deterioration: An application to coatings on steel structures, Rel. Eng. \& Sys. Safety, 92, 1635-1650.

Orcesi, A.D. Chemineau, H. Lin, P.-H. van Gelder, P. van Erp, N. (2016), A Risk Analysis for Asset Management Considering Climate Change, Trans. Res. Proc., $14,105-114$

Park, C. \& Padgett W.J. 2005. Accelerated Degradation Models for Failure Based on Geometric Brownian Motion and Gamma Processes, Lifetime Data Analysis, 11, 511-527.

Ramìrez, P.A. \& Utne, I.B. 2015. Use of dynamic Bayesian networks for life extension assessment of ageing systems, Rel. Eng. \& Sys. Safety 133, 119-136.

Riascos-Ochoa, J. Sànchez-Silva, M. Klutke, G.A. 2016. Modeling and reliability analysis of systems subject to multiple sources of degradation based on Lévy processes, Prob. Eng. Mech., 45, 164-176.

Straub D. 2009. Stochastic modeling of Deterioration Processes through Dynamic Bayesian Networks, J. of Eng. Mech. ASCE 135:1089-1099.

Trappey, A.J.C. Trappey, C.V. Tsao, W.-T. (2012), Engineering Asset Life Span Evaluation Using Logistic Regression, Proc. 7th W. Cong. Eng. Asset Man

Tse, P.W. \& Shen, C. (2013), Remaining Useful Life Estimation of Slurry Pumps Using the Health Status Probability Estimation Provided by Support Vector Machine, Engineering Asset Management-Systems, Professional Practices and Certification, Lecture Notes in Mechanical Engineering.

Van Noortwijk, J.M. 2009. A survey of application of gamma processes in maintenance, Rel. Eng. \& Sys. Safety 94, 2-21. 